\title{
An Electronic Nose for CFRP Bonding safety in aerospace industry: Sensor selection
}

\author{
S. De Vito ${ }^{1}$, G. Fattoruso $^{1}$, E. Massera ${ }^{1}$, M. Miglietta ${ }^{1}$ and G. Di Francia ${ }^{1}$ \\ ${ }^{1}$ UTTP-MDB, ENEA C.R. Portici, P.le E. Fermi, 1, 80055 Portici, Naples, Italy \\ saverio.devito@enea.it
}

\begin{abstract}
:
Lightweight aircrafts, based on composite materials, represent the primary goal of aerospace industry in the close future, allowing considerable savings in aircraft operation costs. Carbon Fiber Reinforced Plastics (CFRP) panels are the basic components for lightweight aircrafts. Their assembly is primarily based on adhesive technologies but, currently, no NDT technique has been validated for ensuring the quality of the bonds while low quality bonding, often caused by surface contamination, would lead to weak bonding with potential airframe failures. Operative needs such as portability, rapid response, versatility make e-noses to represent an interesting solution to develop an ad-hoc tool for surface contamination detection in this scenario. In this work we present the results of a sensor selection procedure for such an e-nose based tool.
\end{abstract}

Key words: Electronic nose, Non Destructive Testing, CFRP Bonding, Artificial Olfaction.

\section{Background and Motivation}

Aerospace industry and research funding players like EU (CleanSky JTI) have devoted significant efforts for financing the investigation of technologies for developing a novel class of lightweight aircrafts. Being able to build up a CFRP based aircraft could allow up to $15 \%$ weight savings on the airframe that, in turn, will generate considerable savings in aircraft operation (fuel, $\mathrm{CO} 2$ emissions) and maintenance [1].

CFRP components are not riveted; their bonding, actually, is based on adhesive technologies. However, research on Non Destructive Testing technologies for quality assessment in CFRP adhesive bonding is still in her infancy and no NDT (Non Destructive Test) technique has been finalized nor validated.

Weak or kissing bonds are a primary safety concern because of the high risk of fatal airframe failures if they occur in primary structures bonding. In particular, surface contamination is one of the main causes of low bonding quality; hydraulic fluids spills, moisture and release agents residuals represent the most common and dangerous contamination sources in the airframe operative life.

Operative needs for a surface contamination detection NDT tool would require detection and classification to be performed within a few seconds, in presence of interferents. Portability is also an important requirement for allowing easy operation in maintenance and repair shops.

Electronic noses (e-noses) are biomimetic gas mixture analysis tools based on array of solid state chemical sensors and on chemometrics algorithm developed in the framework of artificial olfaction [2]. Solid state sensors allow for rapid response and portability whle the use of pattern recognition algorithms allows the enoses to be operated by non skilled personnel. Despite artificial olfaction being still an active research field, e-noses have been successfully been used in a variety of application fields from biomedical sciences to food industry.

For these reasons, E-noses technology represent an interesting solution to develop an ad-hoc NDT tool for surface contamination detection in this scenario. In this work we show the advancements in the screening process of artificial olfaction technologies for the above requirements aiming to the selection of an array of suitable sensors for the development of a portable NDT tool for CFRP pre-bonds quality assessment.

\section{Experimental and Results}

In the framework of project ICARO (In-field composite bonding quality assessment by robotic olfaction), our group is in charge of the development of an electronic nose for surface 
contamination detection and classification. Functional and operative requirements require the capability to detect low quantities (less than $1 \mathrm{~g}$ per $\mathrm{m}^{\wedge} 2$ ) of surface contaminants on CFRP panels within less than $30 \mathrm{sec}$. Contaminants should be present in their liquid phase. Skydrol 500-B, an hydraulic fluid, and Killfrost deicing agent were selected as relevant contaminant for their impact on the quality of CFRP bonded repairs.

One of the main tasks in the design of an artificial olfaction tool is the selection of the sensors that will build up the e-nose sensors array. Sampling system design is also critical. Preliminary investigation resulted in the exclusion of polymer based chemical sensors, for stability reasons. Although they have shown significant sensibility and discrimination capabilities they were affected by poisoning effects caused by the aggressive chemicals under investigation. However, a commercial enose equipped with a polymer sensor array has shown interesting detection and discriminations capabilities of an highly aggressive hydraulic fluid such as the SKYDROL500-B.

In this work we investigated the use of an hybrid array built up by non selective sensors. More specifically we have adopted 2 MOX sensors, 1 Electrochemical sensors, 1 PID sensor and 1 IMS sensor. The use of an integrated IR surface heater has been devised to increase the volatile uptake by the electronic nose sampling system. IMS response were processed to extract significant features (AuC in two different time of flight ranges) from both positive and negative ion spectra so defining 4 virtual sensors.

The sensor array was exposed to the headspace of 15 contaminated CFRP samples and 5 non-contaminated samples.

Contamination ranged from 200 to $500 \mathrm{mg}$ per square meter. The sensors responses were recorded for $60 \mathrm{sec}$ at 1 sample per second rate. Recording took place along ten days. Point by point responses have been used to build up a database that have been used to train and test linear (fisher discriminant based), quadratic, Parzen-like and FFNN (Feed Forward Neural Network) classifiers by using the well known PRTools toolbox [3].

In particular, a randomly chosen $40 \%$ of the entire dataset has been used for training purposes while the remaining samples have been used for testing purposes. Classification performance have been computed by using a tenfold crossvalidation procedure. Percentage of correct classified samples, False positive andFalse Negative rates and the averaged confusion matrix have been used as performance indicators to compare the classifiers performances.

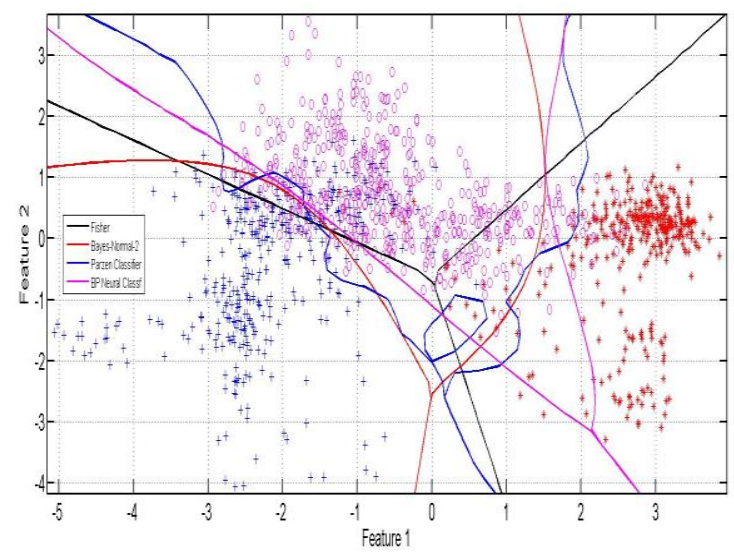

Figure 1: 2D LDA projection of Skydrol contaminated CFRP related e-nose responses (blue crosses), Killfrost contaminated related samples (violet circles) and uncontaminated CFRP related (red stars) samples. Classifiers separation hyperplanes projections have been also drawn.

In figure 1, discrimination capability of the electronic nose are shown by using a 2D LDA projection. Skydrol contaminated CFRP appear to induce a peculiar fingerprint in the response of the sensor array that make them easily discriminated from uncontaminated samples. Discrimination of Skydrol and Killfrost related sample appear only slightly more difficult. Comparison of the performances of the tested classifier shown that the linear classifier provided unsatisfying results while Quadratic, Parzen based and Neural Network classifier provided very similar overall performances. However the quadratic classifiers scored the best overall performances.

Table 1: Samples confusion matrix of Quadratic Classifier

$\begin{array}{lcccc}\text { Estim. Labels } & \text { Skydrol } & \text { Uncontam. } & \text { Killfrost } & \text { Totals } \\ \text { True labels } & & & & \\ \text { Skydrol } & 242 & 2 & 29 & 273 \\ \text { Uncontam. } & 2 & 233 & 9 & 244 \\ \text { Killfrost } & 21 & 14 & 308 & 343 \\ \text { Total } & 284 & 257 & 319 & 860\end{array}$

In Table 1, the averaged samples confusion matrix is reported for the Quadratic classifier. From the confusion matrix it is possible to extract performance indicators as the percentage of total correct classifiers samples that reach $91 \%$. False positive rate is computed as being equal to $4.5 \%$ while false negative rate can be computed as being $2.6 \%$. at the same 
time percentage of Skydrol related samples erroneously classified as Killfrost samples is still significant (10.6\%), the same apply to Killfrost samples erroneously classified as Skydrol contaminated (6.3\%). However, since these figures relate with real time sample by sample classification performance, it is reasonable to explect that a majority voting on a moving average of a limited number of samples may allow to put to zero these misclassifications.

\section{Conclusions}

An hybrid array of chemical sensors has been screened for its detection and discrimination capability of CFRP surface contamination by Skydrol 500-B and Killfrost deicing agent down to $200 \mathrm{mg}$ per square meter. A quadratic classifier trained to discriminate the surface contaminants by analyzing the sensor array combined response has shown interesting performances with its false negative rate being less than $3 \%$.
This preliminary results encourage us to further explore the capability of this setup by analyzing the performance obtainable on long term contaminated samples and the stability of the learned mapping.

\section{Acknowledgements}

This work has been partially funded by CleanSky JTI SFWA demonstrator initiative under the ICARO project grant.

\section{References}

[1] The ENCOMB (Extended Non Destructive Testing for Composite Bonding) project website: http://www.encomb.eu

[2] Pearce, T.C.; Schiffman, S.S.; Nagle, H.T.; Gardner, J.W.; Handbook of Machine Olfaction Electronic Nose Technology, 2003 John Wiley \& Sons

[3] Ferdi van der Heijden, Robert P.W. Duin, Dick de Ridder and David M.J. Tax, John Wiley \& Sons, ISBN 0470090138, 2004. 